Casos C línicos

Arch. Esp. Urol., 58, 4 (354-359), 2005

\section{HEMATOMA SUBEPITELAL DE LA PELVIS RENAL COMO CAUSA DE DEFECTO DE REPLECIÓN EN EL ESTUDIO RADIOLÓGICO DEL TRACTO URINARIO SUPERIOR.}

\author{
$M$ anuel $O$ rtiz $G$ orraiz ${ }^{1}$ y M iguel Angel Campaña \\ G utierrez ${ }^{1,2}$
}

Servicio de Urología ${ }^{1}$. Hospital Universitario Virgen de las $N$ ieves y C línica la Salud ${ }^{2}$. G ranada. España

Resumen.- O BJETIVO: Tras ser atendida una paciente con sospecha de neoplasia de vía urinaria alta, pero que fina Imente fue diagnostica da de hema toma subepitelial de pelvis renal (lesión de Antopol-G oldman), decidimos revisar esta infrecuente patología con el fin de recoger las principales características de esta lesión en base a la bibliografía examinada y nuestra propia experiencia.

MÉTODO S: La paciente se presentó con clínica y datos radiológicos totalmente compatibles con la presencia de tumor urotelial de vía alta, decidiéndose realizar cirugía habitual en estos casos consistente en nefroureterectomía. Entre sus antecedentes solamente destacaba la toma de anticoagulación oral tras presentar cuadro de trombosis venosa profunda.

RESULTADO S: El estudio a natomopatológico de la pieza de nefrectomía concluye con la ausencia de tumor urotelial, y presencia de hematoma subepitelial de pelvis renal que "a posteriori" explican tanto la clínica de la paciente

$M$ anuel 0 rtiz $G$ orraiz

c/ Don Q uijote, $330 \mathrm{H}$

$18008 \mathrm{G}$ ranada. (España)

e-mail: mortig@fundacionhvn.org

Trabajo recibido: 17 de noviembre de 2004 como los hallazgos radiológicos que presentó. N uestro caso se ajusta en muchos puntos a los 27 casos que hemos encontrado publicados en la bibliografía.

DISCUSIÓ N : El hematoma subepitelial de pelvis renal es una lesión benigna, con más predilección por el sexo femenino y que se da fundamentalmente entre la $4 a-6$ a década de la vida. Poco se conoce sobre su etiopatogenia, pero posiblemente se trate de un proceso multifactorial. Tanto clínica como radiológicamente es compatible con la presencia de un tumor urotelial de vía superior, y como tal se ha tratado en la mayoría de los casos publicados. Es necesario conocer esta entidad como posible diagnóstico diferencial de los defectos de repleción en el estudio radiológico del a para to urinario superior para evitar una nefrectomía innecesaria.

Palabras clave: Hematoma subepitelial. Riñón.

Antopol-G oldman.

Summary.- O BJEC TIVES: After taking care of a patient with suspicion of upper urinary tract neoplasia which was finally diagnosed of renal pelvis subepithelial hematoma (Antopol-Goldman lesion), we decided to review such unfrequent pathology with the aim to summarize its main characteristics.

METHODS: The patient presented clinically and radiologically as an upper urinary tract tumor and underw ent nephro ureterectomy. Past history was significant for oral a nticoagula tive trea tment a fter deep vein thrombosis.

RESULTS: Pathologic study of the nephrectomy specimen reported absence of urothelial tumor and presence of a subepithelial hematoma of the renal pelvis, which explained both clinical manifestations and radiological findings. 0 ur case is very similar to the other 27 published in the bibliography.

CON C LUSIO N S: Subepithelial hematoma of the renal pelvis is a benign lesion, more frequent in females, which appears between the fourth and sixth decades of life. Not much is known about its etiopathogenesis, it probably is a multifactorial process. It simulates an upper urinary tract urothelial tumor, both clinically and radiologically, and so is treated in most published cases. It is necessary to know this entity as possible differential diagnosis of upper urinary tract radiological study filling defects to a void non necessary nephrectomies.

Keywords: Subepithelial hematoma. Kidney. Antopol-G oldman. 


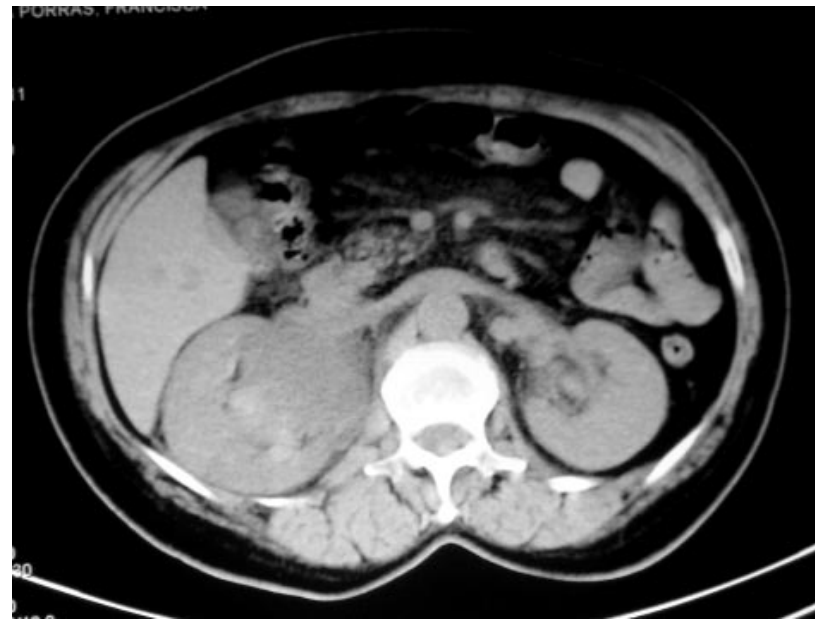

FIG URA 1: Estudio mediante TAC sin contraste i.v. perteneciente a este caso, donde se aprecia ocupación prácticamente total de pelvis renal derecha por material de densidad de partes blandas, con aumento del tamaño de forma global del riñón derecho.

\section{INTRODUCCIÓN}

En 1948, Antopol y Goldman (1) describen por primera vez una serie de siete casos caracterizados por hematuria y defecto de repleción en la pelvis renal detectado mediante estudio urográfico, secundarios a la presencia de hematoma subepitelial de la pelvis renal, confiriendo a éste un aspecto neoplásico. A éste hallazgo lo denominaron lesión de Antopol-G oldman. Presentamos un nuevo informe de esta rara entidad clínica y revisamos la literatura con el fin de recoger aquellos aspectos que la caracterizan.

\section{MATERIAL Y MÉTODOS}

Paciente mujer de 67 años de edad que acude al Servicio de Urgencias refiriendo cuadro de dolor en fosa lumbar derecha, de varios días de evolución y acrecentado en las últimas horas. Igualmente refería mal estado general y astenia. Entre sus antecedentes personales destacamos haber presentado cuadro de trombosis venosa profunda en miembro inferior hace unos meses y en la actualidad en tratamiento con anticoagulación oral, sin otras complicaciones. La paciente no presentaba otro antecedente de interés ni relataba traumatismo previo.

Tras una valoración inicial, comprobándose el mantenimiento de las constantes vitales, los estudios complementarios (analítica de sangre y orina) sólo destacaron una ligera anemia normocítica y microhematuria.

El estudio de imagen se realizó mediante TAC que visualizó defecto de repleción a nivel de la pelvis renal derecha, con diagnóstico radiológico de alta sospecha de tumor urotelial de vía alta (Figuras 1, 2 y 3). Las pruebas de imagen se completaron mediante RM N (Figura 4) que ratificó el diagnóstico de sospecha. La citología de orina fue negativa para malignidad.

Con este diagnóstico, se decide proponer a la paciente cirugía mediante nefroureterectomía derecha por vía de laparotomía media xifopubiana, que acepta.

\section{RESULTADOS}

Durante el acto quirúrgico se evidencia la presencia de trombosis de vena cava abdominal a nivel infrarrenal, llegando esta a la altura de las venas iliacas comunes. Como consecuencia de esta trombosis venosa la paciente presentaba gran dilatación venosa de la red vascular colateral. Tras la liberación del riñón derecho, y al com-

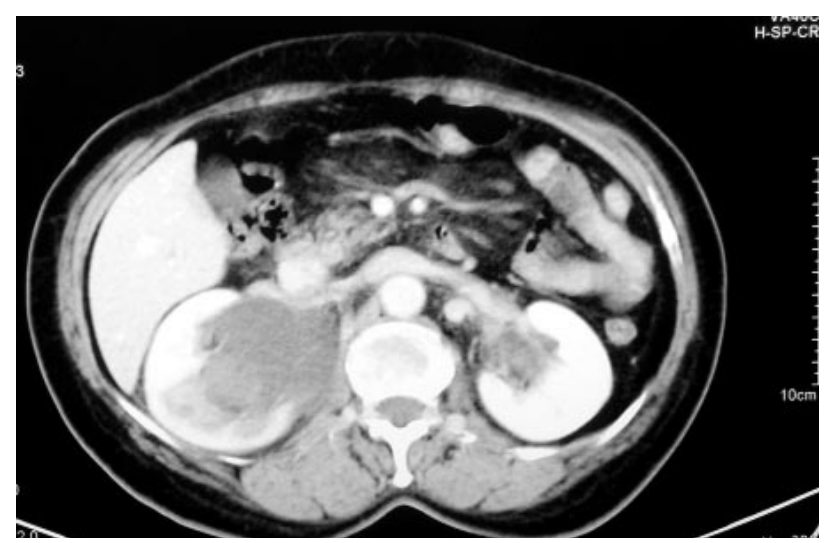

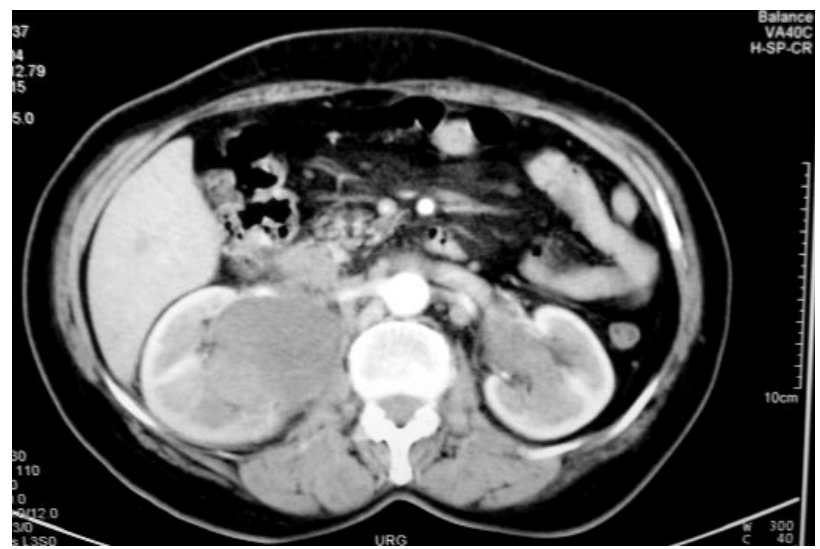

FIG URA 2 y 3: M ismo caso, tras inyección de contraste i.v., no apreciando realce radiológico a nivel de la masa ocupante de pelvis renal, que corresponde a la presencia de hematoma subepitelial de pelvis renal derecha. 
probar un aspecto congestivo de éste, se decide la ligadura y sección del uréter derecho a nivel lumbar, así como estudio histopatológico intraoperatorio de la pieza, que descarta la existencia de tumor urotelial de pelvis, llegándose al diagnostico de hematoma subepitelial a nivel de la pelvis renal, por lo que se decide concluir la intervención con preservación del remanente ureteral derecho. El análisis anatomopatológico definitivo confirmó la lesión de Antopol-Goldman. El postoperatorio cursó sin incidencias, encontrándose actualmente la paciente sin problemas tras seis meses de la cirugía.

\section{DISCUSIÓN}

Ya en la primera descripción que se publicó de esta infrecuente entidad, Antopol y Goldman (1) señalan la dificultad de su diagnóstico preoperatorio, realizándose en la mayoría de casos nefrectomía ante la alta sospecha de tumor urotelial de vía urinaria superior, no siendo hasta después del examen anatomopatológico de la pieza quirúrgica cuando se detectó el hema toma subepitelial como el causante del cuadro descrito.

Este hecho se recoge en la casi práctica totalidad de los casos publicados de esta lesión, un total de 28 incluyendo el aquí presentado. Las características clínicas, radiológicas y patológicas descritas en estos casos y que definirían a este cuadro clínico se recogen en la Tabla I.

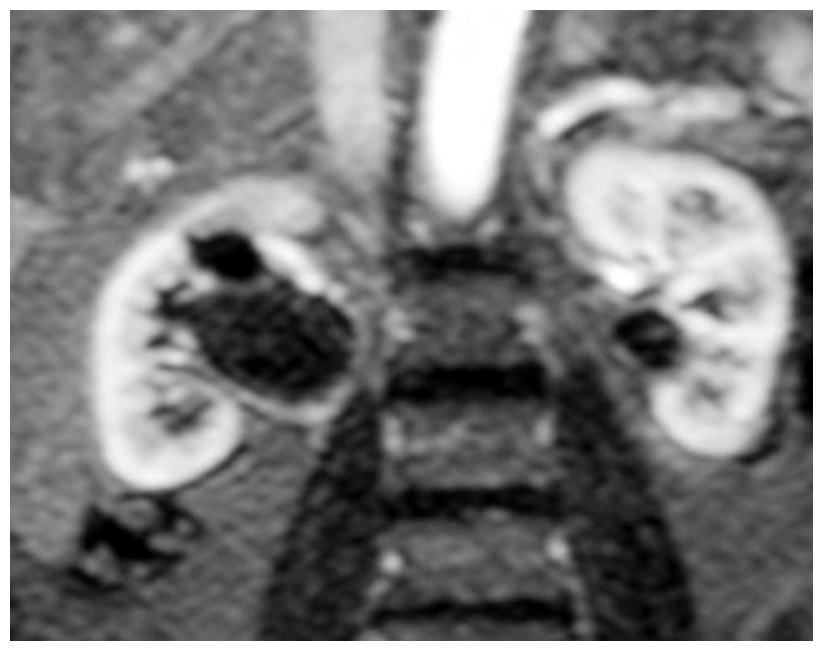

FIG URA 4: M isma paciente, estudio mediante RM N potenciada con $G$ adolinio i.v. (secuencia T2) que muestra buena diferenciación córtico-medular y defecto de repleción en pelvis renal derecha, que corresponde a existencia de hemorragia subepitelial a este nivel.
Como se puede deducir de esta tabla, el caso típico afecta a una mujer en la 4ạ-6a década de su vida, que se presenta con dolor en flanco y hematuria, con un defecto de repleción en la pelvis renal detectado en estudios radiológicos, siendo estas dos últimas características prácticamente constantes en todos los casos descritos (2).

La causa de esta lesión no ha sido aún aclarada, pero podemos pensar que se trata de un cuadro de origen multifactorial. De esta forma, parece que se pueden establecer algunos factores predisponentes (relacionados con el riñón), asimismo como una serie de factores precipitantes que facilitarían el desarrollo del sangrado a nivel subepitelial. Así, entre los primeros se ha destacado la más frecuente afectación del riñón derecho (57\%) asociado a una mayor hipermovilidad (2) de éste. También se han encontrado anomalías congénitas de la vascularización renal (angiomas corticales) o de la pelvis renal con presencia de hemangiomas, así como vasos renales aberrantes (1), con o sin infarto cortical asociado. El estudio histopatológico de la pieza ha revelado riñones de características normales en algunos casos (3), pero también de tamaño aumentado en relación con una hiperemia renal (4), o bien casos de alteraciones congénitas como pelvis extrarrenal, pelvis y uréter doble, lobulación fetal aberrante e inserción anómala del ureter (3). En la superficie del revestimiento urotelial se han encontrado múltiples petequias, zonas denudadas de urotelio $(2,5)$ así como lesiones ulcerosas $(1,3,6)$ cuyo significado etiológico es incierto y más bien pudiera tratarse de estigmas secundarios a la presencia del hematoma subepitelial. 0 tro hallazgo, descrito igualmente en nuestro caso, ha sido la presencia de venas estásicas (4), que pudiera favorecer el sangrado por detención crónica del flujo sanguíneo y rotura espontánea de capilares subepiteliales, si bien este extremo no ha sido demostrado ni en nuestro paciente ni en otros previos. Esta condición, con la presencia de hematoma subepitelial en el estudio histopatológico de la pelvis renal no es exclusiva del riñón, sino que ha sido descrita con relación a otros órganos como esófago (4), estómago y epitelio gingival.

En cuanto a los factores precipitantes de esta lesión, se ha dado gran importancia al traumatismo renal, incluso de grado leve como podría suponerse en un cambio brusco de postura, aunque este antecedente sólo se ha podido establecer con seguridad en tres casos $(1,7)$, existiendo en otros casos publicados antecedente de biopsia renal percutanea unos días antes (3) y parto vaginal sin complicaciones en las dos semanas previas (4). 0 tros factores relacionados han sido la hipertensión arterial (3), litiasis renal (4), consumo de medicación anticoagulante 
(8) y consumo crónico de analgésicos con capacidad antiagregante plaquetaria $(5,9)$ cuyo abuso de forma experimental se ha asociado a una proliferación de la microvascularización renal (5). No han sido descritas coagulopatías en estos pacientes, ni tampoco la presencia de sangrados espontáneos a otros niveles, aunque en ningún caso se descartó este extremo mediante estudios específicos de hemostasia. Este hecho haría pensar en la presencia de un factor local en la propia pelvis renal como principal causante de esta lesión. Igualmente tampoco se han descrito factores infectivos ni lesiones mediadas por el sistema inmune del tipo de las vasculitis en los casos publicados previamente.

Desde el punto de vista clínico, destacan la presencia de hematuria macroscópica y dolor en flanco ipsilateral en cerca del $80 \%$ de todos los casos, habitualmente de presentación súbita. La intensidad de la hematuria es tal que en ocasiones, ante la dificultad para la micción por la presencia de coágulos vesicales, se ha requerido el sondaje vesical para la evacuación de éstos (6), aunque por lo general es leve. La exploración suele ser anodina, sin signos de repercusión hemodinámica por el sangrado, aunque si puede ser dolorosa la percusión de la fosa lumbar del lado afectado.

Las pruebas complementarias basadas en los parámetros hematológicos aportan escasa información, ya que no suele existir descenso destacable del hematocrito, alteraciones de la función renal con ascenso de la creatinina, ni trastornos de los valores de coagulación sanguínea. Si podemos evidenciar, en aquellos pacientes que no presenten historia de hematuria, hematíes en el sedimento de orina. También se ha descrito la presencia de intensa proteinuria que desapareció tras nefrectomía parcial (5). La citología de orina es constantemente negativa, siempre y cuando no coexista un tumor urotelial a cualquier nivel del urotelio.
La cistoscopia puede descartar el origen vesical de la hematuria, así como evidenciar en algunos casos el eyaculado hematúrico a través del orificio ureteral ipsilateral (6). La ureteroscopia puede resultar dificultosa por el continuo sangrado, la presencia de coágulos en la vía urinaria y la sobreelevación de la mucosa urotelial por la hemorragia subepitelial (6). Sin embargo es posible que la ureteropieloscopia retrógrada pueda ayudar en algunos casos dudosos donde se logre establecer la sospecha de hematoma subepitelial.

Todos los autores están de acuerdo en la importancia de los estudios de imagen para llegar a un diagnóstico preoperatorio de la lesión, si bien esto es un extremo excepcional en la literatura revisada (7). La hemorragia subepitelial a nivel de la pelvis renal se expresa como una sobreelevación del epitelio, lo que es responsable del aspecto tumoral en las pruebas de imagen (3), fundamentalmente en la urografía intravenosa. Ésta imagen radiológica estará en función de la extensión del hematoma, el cual puede afectar desde únicamente la pelvis renal o extenderse hacia cálices y uréter proximal (4). La U.I.V. puede también detectar la presencia de uropatía obstructiva o de anulación funcional de la unidad renal afectada (3). La pielografía ascendente no aporta datos con respecto a la urografía (4) a no ser que se trate de un riñón no funcionante. Puesto que las imágenes urográficas son inespecíficas (Tabla II), es necesaria la combinación de esta prueba con estudios de mayor calidad en la imagen. Creemos que la ecografía no añade beneficio diagnóstico en estos casos, ya que los procesos descritos informaban de efecto masa de ecogenicidad heterogénea (2), tratándose ésta de una lesión ultrasonográfica muy inespecífica. Así, sería el estudio mediante TAC con contraste intravenoso el que puede revelar más datos, y cuyos signos radiológicos más se han estudiado en la bibliografía revisada. Justamente, se puede apreciar una masa lobulada y bien definida, ocupante de la luz en pel-

\section{TABLA I. CARAC TERÍSTICAS CLÍN IC O RADIO LÓ GICAS DE LO S 28 CASOS PUBUCADO S DE HEMATO MA} SUBEPITEUAL DE PELVIS REN AL

\begin{tabular}{|c|c|c|c|c|c|c|c|}
\hline Edad (años) & Sexo & Riñón & Dolor & Hematuria & $\begin{array}{l}\text { Antec. } \\
\text { Trauma }\end{array}$ & Radiología & Tratamiento \\
\hline Media: 46 & Mujer: 18 & D: 16 & Sí: 20 & MacroH:22 & No: 25 & Defecto repleción: 16 & N efrectomía: 24 \\
\hline Rango: 28-84 & Hombre: 10 & $\mathrm{I}: 12$ & No: 8 & MicroH: 6 & Sí: 3 & $\begin{array}{l}\text { Dilatación vía urinaria: } 6 \\
\text { Efecto masa: } 3 \\
\text { ND: } 3\end{array}$ & $\begin{array}{l}\text { N efrectomía parcial: } 2 \\
\text { Tto. conservador: } 1 \\
\text { Pieloplastia: } 1\end{array}$ \\
\hline
\end{tabular}

MacroH: macrohematuria; MicroH: microhematuria; N D: no documentado; Tto: tratamiento. 
vis renal, y con densidad de partes blandas (35-45 HU) que no se realza tras la administración de contraste intravenoso $(10,11)$, al igual que sucede en la serie de imágenes aportadas en este caso. La especificidad de la prueba para el diagnóstico del hematoma subepitelial ha sido demostrada en algún caso aislado (7). Pueden igualmente detectarse coágulos en el interior de la vía urinaria. Creemos que la angiografía en menor grado, y sobre todo el estudio mediante RM pueden permitir un diagnóstico más exacto en aquellos casos que el TAC no permita un dictamen exacto, sin existir hasta el momento documentación acerca de las imágenes de RM en relación con el hematoma subepitelial, no habiendo permitido su realización en nuestro caso el diagnóstico preoperatorio de hematoma.

A I revisar la literatura observamos que en un $67 \%$ de los casos, el diagnóstico de sospecha era un tumor urotelial o renal en base a los hallazgos clínico-radiológicos. El resto de casos se relacionaron con: hematoma renal, hematoma subepitelial de inicio, angioma renal, estenosis de la UPU e hidronefrosis con anulación funcional del riñón.

Un factor no recogido en la literatura es la historia natural de esta lesión. No está claro si se trata de una hemorragia aguda 0 si por el contrario se produce de una forma subaguda o crónica. Aunque existen casos de pacientes con hematuria y dolor de meses de evolución (4), no se ha podido evidenciar la evolución que sigue esta lesión desde su instauración hasta el desarrollo de clínica (dolor y/ o hematuria). Si se ha recogido el control evolutivo radiológico tras su diagnóstico en un paciente tratado de forma conservadora (7) y que más adelante se comentará.

Llama la atención, que en ninguno de los casos aportados en la literatura, la lesión de Antopol-Goldman se asoció a la presencia real de neoplasia a nivel renopiélica una vez estudiada la pieza quirúrgica.

El tratamiento, conceptualmente, debería ser conservador, con buenos resultados mediante reposo absoluto y analgésicos en un caso descrito en el que se actuó de esta forma ante la alta sospecha de hemorragia subepitelial de pelvis renal mediante las pruebas de imagen (7). Sin embargo, la dificultad diagnóstica que supone la identificación preoperatoria de esta lesión, así como la sospecha de encontrarnos ante un paciente con una neoplasia urotelial, hace que la casi totalidad de los 28 casos descritos en la literatura hayan requerido intervención quirúrgica de forma inmediata o diferida. Por otro lado, aunque el diagnóstico preoperatorio permita inicialmente un manejo conservador, la persistencia en ocasiones de hematuria incoercible puede llevar a requerir la intervención quirúrgica (6), debiéndose optar en estos casos por la nefrectomía parcial como técnica quirúrgica que ha demostrado buenos resultados con remisión de la hematuria tras la intervención (5). Igualmente se recoge en la literatura un caso de hematoma subepitelial de la pelvis renal que comportándose como una estenosis de la unión pieloureteral tuvo buena respuesta al tratamiento median-

TABLA II. CAUSAS DE DEFECTO DE REPLECIÓN EN EL ESTUDIO RADIO LÓ GICO DEL TRACTO URIN ARIO SUPERIO R

\section{Malignas}

Carcinoma de células transicionales

A denocarcinoma renal

Carcinoma metastásico

Carcinoma de células escamosas

\section{No malignas}

\author{
Cálculos \\ Tuberculosis \\ Pólipo fibroepitelial \\ Papiloma invertido \\ Adenoma nefrogénico \\ Infección (bezoar) \\ Coágulos \\ Hematoma subepitelial (lesión de A ntopol-G oldman) \\ Endometriosis \\ A miloidosis \\ Desprendimiento de papila \\ Compresión extrínseca
}


te pieloplastia desmembrada y que el autor recomienda como alternativa ante esta lesión (4).

Tras el tratamiento conservador mediante reposo y analgésicos, se puede comprobar la resolución del cuadro con la realización de estudios radiológicos, sin existir actualmente ningún protocolo ampliamente aceptado para el manejo y seguimiento de este tipo de pacientes que sufren cuadros de hematuria por lesiones benignas a nivel del tracto urinario superior y que se pudieran manejar de inicio de forma conservadora. Si parece que sería importante el no retrasar el primer control más allá de las 3-4 primeras semanas, con el fin de detectar precozmente la persistencia de esta lesión y por tanto la toma de decisiones en cuanto al mantenimiento de la actitud conservadora o el paso a un manejo mas intervensionista. En el único caso descrito en el que se opto únicamente por el tratamiento conservador, los controles posteriores a los 6 días y 3 semanas mediante UIV y TAC confirmaron la total desaparición del defecto de repleción en pelvis renal (7).

Por último añadir que no ha sido descrito ningún caso de bilateralidad sincrónica ni metacrónica, así como de fallecimiento por esta lesión. La principal consecuencia que puede tener el paciente mal diagnosticado de inicio será la perdida de una unidad renal por lo demás posiblemente sin ninguna otra patología asociada.

\section{CONCLUSIÓN}

Podríamos resumir que el hematoma subepitelial de pelvis renal es una lesión benigna con características radiológicas de malignidad y que requiere un alto índice de sospecha para su diagnóstico mediante pruebas de imagen. Ante su sospecha se debería actuar de forma conservadora, de forma similar a otros procesos sangrantes renales autolimitados, con controles radiológicos de la evolución. En caso de duda diagnóstica podríamos intentar la cirugía conservadora sobre el riñón afectado, dejando la nefrectomía para aquellos casos que acuden a quirófano con el diagnostico de tumor urotelial. Como en otras entidades nosológicas, para llegar a su diagnóstico es necesario conocer los posibles procesos patológicos que cursan con defecto de repleción a nivel de la vía urinaria superior.

\section{BIBUOGRAFIA y LECTURAS \\ RECOMENDADAS (*lectura de interés y \\ **lectura fundamental)}

*1. ANTOPOL, W.; GOLDMAN, L.: "Subepithelial hemorrhage of renal pelvis simulating neoplasm". Urol. Cutan. Rev., 52: 189, 1948.

2. KIM, S.J.; JUN, H.S.; CHUNG, D.Y. y cols.: "Subepithelial hematoma of the renal pelvis simulating neoplasm (Antopol-Goldman lesion)". Urol. Int., 59: 260, 1997.

**3. VILLAR PASTOR, C.M.; LÓPEZ BELTRÁN, A.; ÁLVAREZ KINDELÁN, J. y cols.: "Hemorragia subepitelial de pelvis renal (lesión de Antopol-Goldman). Presentación de cuatro casos y revisión de la literatura”. Actas Urol. Esp., 24: 805, 2000.

**4. ICZKOWSKI, K.A.; SWEAT, S.D.; BOSTWICK, D.G.: "Subepithelial pelvic hematoma of the kidney clinically mimicking cancer: report of six cases and review of the literature". Urology, 53: 276, 1999.

5. LEVITT, S.; WAISMAN, J.; DeKERNION, J.: "Subepithelial hematoma of the renal pelvis (AntopolGoldman lesion): a case report and review of the literature". J. Urol., 131: 939, 1984.

6. OZA, K.N.; REZVAN, M.; MOSER, R.: "Subepithelial hematoma of renal pelvis (AntopolGoldman lesion)". J. Urol., 155: 1032, 1996.

*7. RODRÍGUEZ ALONSO, A.; GONZALEZ BLANCO, A.; CESPÓN OUTEDA, E. y cols.: "Hematoma subepitelial de pelvis renal y uréter: lesión de AntopolGoldman”. Actas Urol. Esp., 26: 133, 2002.

8. VIAMONTE, M.; ROEN, S.A.; VIAMONTE, M.Jr. y cols.: "Subepithelial hemorrhage of renal pelvis simulating neoplasm (Antopol-Goldman lesion)". Urology, 16: 647, 1980.

*9. DEMIRKAN, N.C.; TUNCAY, L.; DÜZCAN, E. y cols.: "Subepithelial haematoma of the renal pelvis (Antopol-Goldman lesion)". Histopathology, 35: 282, 1999.

10. KOSSOL, J.M.; PATEL, S.K.: "Suburothelial hemorrhage: the value of preinfusion computed tomography”. J. Comput. Assist. Tomogr., 10: 157, 1986.

11. MILlER, V.; WITTEN, D.M.; SHIN, M.S.: "Computed tomographic findings in suburothelial hemorraghe". Urol. Radiol., 4: 11, 1982. 